\title{
Hepatitis C Positive Organ Donation in Heart Transplantation
}

\author{
Hasan K. Siddiqi ${ }^{1} \cdot$ Kelly H. Schlendorf ${ }^{1}$ \\ Accepted: 18 October 2021 / Published online: 10 November 2021 \\ (C) The Author(s), under exclusive licence to Springer Nature Switzerland AG 2021
}

\begin{abstract}
Purpose of Review To summarize outcomes to date, as well as important considerations and unanswered questions related to the use of hepatitis $\mathrm{C}$ virus (HCV) positive donors for heart transplantation.

Recent Findings Outcomes from single-center studies and registry data to date suggest that among patients who develop donor-transmitted HCV after heart transplantation, direct-acting antiviral therapies (DAAT) are effective and well-tolerated, and that short-term survival is similar to that of patients transplanted with HCV - donors.

Summary In an era marked by increasing numbers of HCV positive deceased donors and a growing imbalance between the demand and supply of donor hearts, utilization of $\mathrm{HCV}+$ donors is a feasible strategy to expand the donor pool and reduce waitlist times. Ongoing work is needed to clarify longer-term outcomes with the use of this strategy.
\end{abstract}

Keywords Heart transplantation $\cdot$ Hepatitis $\mathrm{C}$ virus $\cdot$ Direct-acting antiviral therapies $\cdot$ Donor pool

\section{Introduction}

Heart transplantation (HT) offers a life-saving treatment for patients with advanced heart failure. With an estimated 6 million adults in the USA living with heart failure [1], there is a growing population of patients with advanced heart failure who may need HT for long-term survival. However, only 3500 HT are performed annually in the USA [1], a number limited primarily due to an insufficient supply of acceptable donor hearts. The growing imbalance between the demand and supply of donor hearts has resulted in extended waiting times on an evergrowing heart transplant wait list. Identification of strategies that expand the donor pool is critical.

The opioid epidemic in the USA has burgeoned over the past decade and been accompanied by a sharp increase in hepatitis $\mathrm{C}$ virus (HCV) transmission due to the use of shared, non-sterile needles. According to data from the Centers for Disease Control, the number of acute HCV infections reported in 2019 was 4,136 compared to 1,778 in 2012, representing

This article is part of the Topical Collection on Thoracic

Transplantation

Kelly H. Schlendorf

kelly.h.schlendorf@vumc.org

1 Division of Cardiology, Vanderbilt University Medical Center, 1215 21, St Avenue South, Suite 5307, Nashville, TN 37232, USA a $132 \%$ increase in reported incident HCV cases [2]. Due to significant under-reporting and diagnosis of $\mathrm{HCV}$, actual new infections are estimated to be $>50,000$ in 2019 [2] (Fig. 1). Furthermore, there has been a continued rise in deaths related to opioid and other drug overdoses, with over 70,000 such deaths in 2019 [3] (Fig. 2). The COVID-19 pandemic has accentuated this trend, with $>10,000$ excess deaths related to drug overdoses during 5 months of the pandemic compared to pre-pandemic levels [4]. Younger age groups have been especially affected by this public health emergency, with adults aged 35-44 representing the age group with the highest rate (40.5 per 100,000 individuals) of drug overdose deaths in 2019 [3]. As a direct result of the opioid epidemic, persons deceased secondary to intravenous drug overdose and with a high prevalence of $\mathrm{HCV}$ infection now represent a growing proportion of the donor pool. Moreover, recent experience suggests that in the contemporary era, HT using these donors should routinely be considered [5•]. Herein, we briefly review outcomes to date and future directions.

\section{Novel Hepatitis C Therapies-Dawn of a New Era}

Contemporary discussion about $\mathrm{HCV}$ is best framed in the context of recent therapeutic advances. In the "old era" (1980s-2011), interferon and ribavirin were 


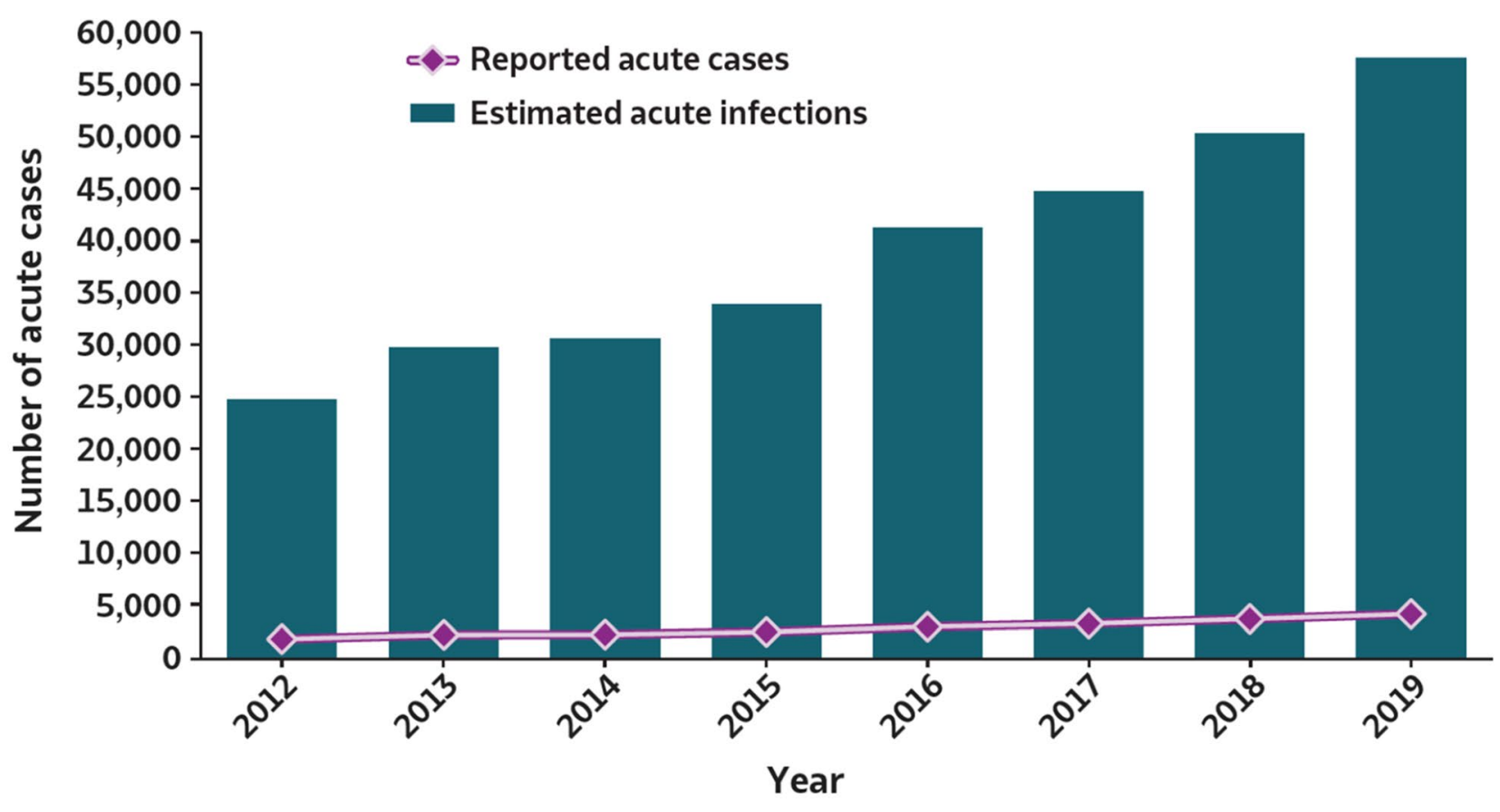

\begin{tabular}{|c|c|c|c|c|c|c|c|c|}
\hline Acute Hepatitis C & $\mathbf{2 0 1 2}$ & $\mathbf{2 0 1 3}$ & $\mathbf{2 0 1 4}$ & $\mathbf{2 0 1 5}$ & $\mathbf{2 0 1 6}$ & $\mathbf{2 0 1 7}$ & $\mathbf{2 0 1 8}$ & $\mathbf{2 0 1 9}$ \\
\hline Reported acute cases & 1,778 & 2,138 & 2,194 & 2,436 & 2,967 & 3,216 & 3,621 & 4,136 \\
\hline Estimated acute infections & 24,700 & 29,700 & 30,500 & 33,900 & 41,200 & 44,700 & 50,300 & 57,500 \\
\hline
\end{tabular}

Source: CDC, National Notifiable Diseases Surveillance System.

"The number of estimated viral hepatitis infections was determined by multiplying the number of reported cases that met the classification criteria for a confirmed case by a factor that adjusted for underascertainment and underreporting. The $95 \%$ bootstrap confidence intervals for the estimated number of infections are displayed in the Appendix.

Fig. 1 Burden of reported and estimated hepatitis C virus infections from 2012 to 2019. https://www.cdc.gov/hepatitis/statistics/2019surveillance/Figure3.1.htm

the mainstay of HCV therapy, despite often intolerable side effect profiles, prolonged lengths of treatment, and limited efficacy [6]. In 2011, the "new era" of HCV therapy began when direct-acting antiviral therapies (DAAT) were shown to be safe and efficacious in curing $\mathrm{HCV}$ with sustained virologic response rates greater than $90 \%$. The newest of these agents, consisting nowadays of combinations of NS3/4A protease inhibitors and NS5A/B polymerase inhibitors-absent interferon or ribavirin-inhibit viral replication and infection with minimal side effects and cure rates $>98 \%$ [7-10]. While some DAAT regimens are HCV genotype specific, pangenotypic DAAT are now available, providing an important tool in scenarios where rapid genotyping may not be possible.

\section{Hepatitis C Positive Organs in Heart Transplant Recipients-the Old Era}

Studies of organ transplantation from HCV positive donors to HCV naïve recipients in the "old era" demonstrated a variable but unacceptably high rate of HCV transmissibility $[11,12]$, associated with poor outcomes. A retrospective study of $34 \mathrm{HCV}$ naïve HT recipients showed a threefold higher risk of mortality and 2.8-fold greater risk of coronary allograft vasculopathy (CAV) among patients who received $\mathrm{HCV}+$ organs compared to those who received HCV - organs [13]. In another study, $261 \mathrm{HT}$ recipients of $\mathrm{HCV}+$ donors demonstrated $50 \%$ lower survival at 1,5 , and 10 years compared to recipients of $\mathrm{HCV}$ - donor hearts, with significantly higher rates of mortality related to viral hepatitis or liver failure, as well as CAV [14]. It was in this context 


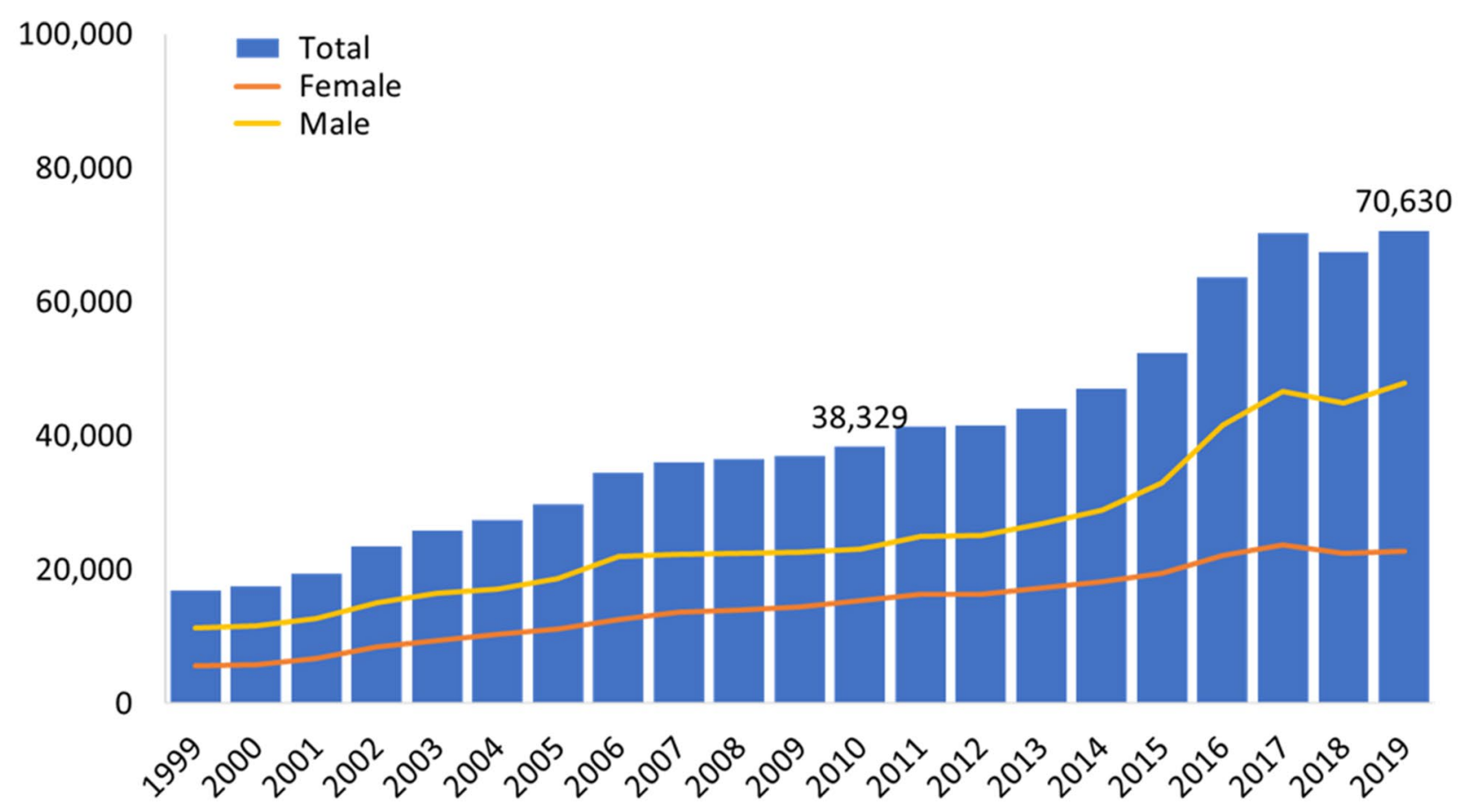

*Includes deaths with underlying causes of unintentional drug poisoning (X40-X44), suicide drug poisoning (X60-X64), homicide drug poisoning (X85), or drug poisoning of undetermined intent (Y10-Y14), as coded in the International Classification of Diseases, 10th Revision. Source: Centers for Disease Control and Prevention, National Center for Health Statistics. Multiple Cause of Death 1999-2019 on CDC WONDER Online Database, released 12/2020.

Fig. 2 Annual drug-related overdose deaths in the USA from 1999 to 2019. https://www.drugabuse.gov/drug-topics/trends-statistics/overdosedeath-rates

during the "old era" that $\mathrm{HCV}+$ donor organs were generally considered unsuitable for HT.

\section{Hepatitis C Positive Organs in Heart Transplant Recipients-the New Era}

Following on the heels of several promising pilot studies transplanting HCV nucleic acid amplification test positive (NAT +) organs in kidney, lung, and liver transplantation in the "new era" [15-17], Baylor University published the first reported case of HT using a HCV NAT + donor in 2017 [18]. The patient, who was HCV naïve at transplant and developed donor-transmitted HCV (dt-HCV) post-HT, was treated with sofosbuvir/velpatasvir and demonstrated sustained virologic response at 12 weeks after completion of DAAT (SVR12).

Shortly thereafter, Vanderbilt published the first case series describing early outcomes in HT utilizing HCV $\mathrm{NAT}+$ donors in HCV naïve recipients [19]. Thirteen patients underwent HT using HCV + donor hearts (11/13 $\mathrm{NAT}+, 2 / 13 \mathrm{Ab}+/ \mathrm{NAT}-$ ), with 9/13 (69\%) developing dt-HCV. HCV therapy was initiated as an outpatient for those with dt-HCV - patients who acquired HCV genotype 1 were treated with 12 weeks of ledipasvir/sofosbuvir, while patients with HCV genotype 3 received 12 to 24 weeks of sofosbuvir/velpatasvir. All but one (89\%) patient with dtHCV completed DAAT without limiting side effects and with subsequent demonstration of SVR12. One patient died of a pulmonary embolism while undergoing DAAT. While this report was limited by its single-center nature, small sample size, and absence of a control group, it nonetheless demonstrated the potential promise afforded by HCV NAT + donors in the "new era."

Following these first experiences with DAAT in HCV NAT + HT, multiple other centers have reported their experiences (Table 1), some pursuing preemptive HCV treatment whereby DAAT are initiated after confirmation of dt-HCV in recipients, and others pursuing a prophylactic approach whereby DAAT are initiated perioperatively in an effort to prevent $\mathrm{HCV}$ transmission.

Woolley and colleagues conducted a trial of 4 weeks of prophylactic sofosbuvir/velpatasvir in HCV NAT + organ transplantation, administered within a few hours of surgery [20]. Thirty-six lung transplant and 8 heart transplant patients were included, with $95 \%$ showing evidence of $\mathrm{HCV}$ viremia immediately after transplantation. Among those with 6-month follow-up at time of publication, $100 \%$ of patients demonstrated SVR at 4 and 24 weeks after 


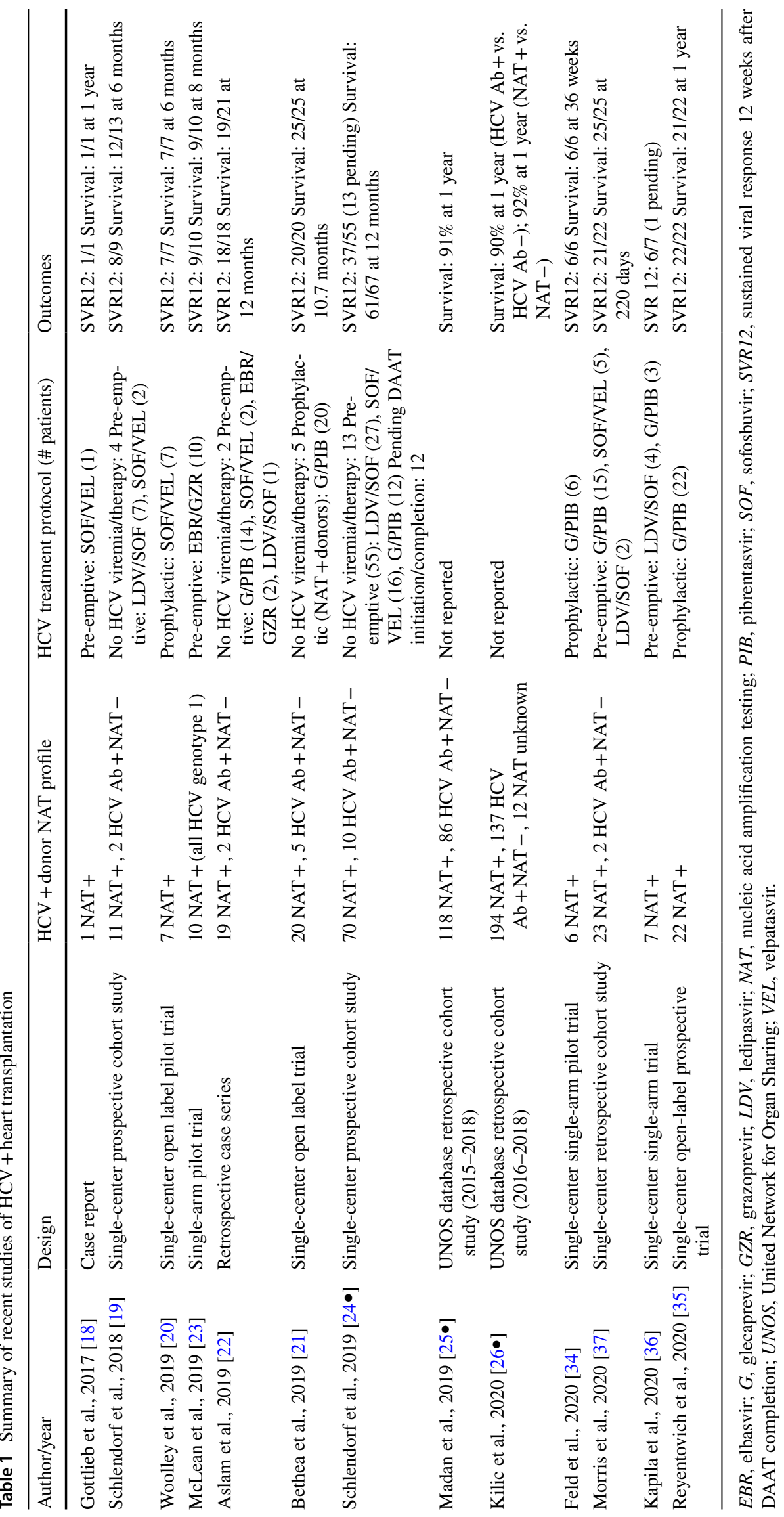


completing therapy. No significant treatment-related serious adverse events were noted. In the HT cohort, graft survival was $100 \%$ at 6 months, not significantly different compared with a group of $\mathrm{HCV}-\mathrm{HT}$ recipients. There was also no significant difference in incident acute cellular rejection between $\mathrm{HCV}+$ and $\mathrm{HCV}-\mathrm{HT}$ recipients. This study was the first to demonstrate efficacy of prophylactic pan-genotypic DAAT in patients undergoing HT with HCV $\mathrm{NAT}+$ donor hearts.

The use of prophylactic DAAT in HCV + HT was further investigated by Bethea and colleagues [21]. Their study included 20 recipients of HCV NAT + donors and

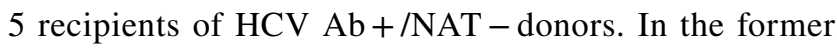
group, glecaprevir/pibrentasvir was started just prior to HT and continued for 8 weeks post-HT. Patients in this group had rapid clearance of virus (median 3.5 days post-HT), and all achieved SVR12. Patient allograft survival was $100 \%$ at 10 months of follow-up.

Among studies pursuing a preemptive HCV treatment approach, Aslam et al. described $21 \mathrm{HCV}+\mathrm{HT}, 19$ from NAT + donors and 2 from NAT - donors [22]. Similar to other studies, recipients of non-viremic (NAT -) donors did not show evidence of dt-HCV, whereas the 19 recipients from viremic (NAT +) donors did. The use of induction immunosuppressive therapies in $13(62 \%)$ patients was not associated with HCV viral load at 2 weeks post-HT. A variety of preemptive DAAT regimens, including glecaprevir/ pibrentasvir ( $74 \%$ of patients), sofosbuvir/velpatasvir (11\% of patients), elbasvir/grazoprevir (11\% of patients), and ledipasvir/sofosbuvir (5\% of patients) were initiated on hospital discharge in $89 \%$ of patients and during index hospitalization for 2 patients. Regardless of DAAT regimen, all patients demonstrated $100 \%$ clearance of HCV viremia and $100 \%$ SVR12. One patient in the HCV NAT + group died of graft failure potentially related to antibody-mediated rejection (although exact cause was not identified), 64 days after completing DAAT (sofosbuvir/velpatasvir). In the safety assessment, $32 \%$ of viremic patients developed transaminitis after HT but prior to DAAT, with resolution of transaminitis in all patients by time of last follow-up [22].

In another, smaller trial, $10 \mathrm{HCV}$ naïve patients underwent HT using HCV genotype 1 cardiac allografts [23]. All patients had detectable HCV viremia by post-transplant day 3 , at which time a 12-week course of daily elbasvir/grazoprevir therapy was initiated (preemptive strategy). Ninety percent of recipients had undetectable HCV NAT within 4 weeks of starting therapy, while one participant had persistent viremia at that time point, prompting 4 additional weeks of DAAT.

In the largest single-center prospective analysis of HT using HCV NAT + donors and a preemptive treatment strategy, the Vanderbilt group followed up its initial report [19] with outcomes in $80 \mathrm{HT}$ using HCV Ab+hearts, 70 of which were recovered from viremic donors and 10 from nonviremic donors [24•]. No recipients of non-viremic donors developed dt-HCV. Of the 70 recipients of viremic donors, 67 (95.7\%) developed dt-HCV. Treatment with DAAT, generally initiated a median of 55 days post-HT, was well tolerated and yielded SVR in all treated patients. Within the cohort with dt-HCV, 1-year patient survival was $90.4 \%$, not significantly different compared with patients who received transplants from $\mathrm{HCV}$ - donors during the same period at Vanderbilt. While recipients of viremic donors had significantly more primary graft dysfunction (PGD) compared to recipients of non-viremic donors $(13.7 \%$ vs. $3.1 \%$, $p=0.002$ ), there were no significant differences in rates of acute rejection requiring treatment or CAV [24•].

Two separate registry studies have added to the findings from single-center experiences. An analysis of the UNOS registry examining survival and PGD by donor HCV status among 6,864 HT recipients transplanted between 2015 and 2018 demonstrated 1-year survival of $91 \%$, with no significant differences based on whether the donor was HCV naïve $(n=6,660)$, HCV Ab $+\mathrm{NAT}-(n=86)$, or $\mathrm{HCV}$ NAT $+(n=118)$ - this finding persisted even after adjustment for recipient age, gender, UNOS status 1A, blood group $\mathrm{O}$, body mass index, inotrope support, left ventricular assist device support, and donor age, gender, ischemic time, and cause of death [25•]. Rates of PGD at 90 days were similar between all three groups in raw and adjusted analyses, as were rates of acute rejection requiring treatment.

In a separate analysis of the UNOS registry, Kilic and colleagues examined HT outcomes based on donor HCV $\mathrm{Ab}$ status, with a sensitivity analysis based on donor NAT status, for HT performed between 2016 and 2018 [26•]. Of the 7889 isolated HT performed during this time frame, 343 (4.4\%) utilized HCV Ab + donor hearts (194 NAT +, 137 NAT - , 12 NAT status unknown) at 36 unique centers. The percentage of transplant centers performing $\mathrm{HCV}$ $\mathrm{Ab}+$ transplants increased from $8.5 \%$ in 2016 to $29.4 \%$ in 2018. For the primary outcome of 1-year survival, there was no significant difference between recipients of $\mathrm{HCV}$ $\mathrm{Ab}+$ versus $\mathrm{Ab}-$ donors, both before and after propensity matching. Adjustment for clinically relevant donor and recipient characteristics did not alter this result, with an adjusted hazard ratio of 1.05 (95\% confidence interval $0.70-1.58, p=0.80$ ) for 1 -year post-transplant survival in the total population. There were no differences in 1-year drug-treated rejection, new-onset post-operative dialysis, post-operative stroke, and length of stay between the groups. In examining outcomes stratified by NAT status, 1-year recipient survival was not statistically different between recipients of $\mathrm{HCV} \mathrm{Ab}+\mathrm{NAT}+$ and $\mathrm{HCV} \mathrm{Ab}+\mathrm{NAT}-$ donors, or between recipients of $\mathrm{HCV}+\mathrm{NAT}+$ and $\mathrm{HCV}-$ donors. Rates of the secondary 
outcomes including rejection, dialysis, and stroke were also similar between the different NAT groups [26•].

While a number of studies have explored patient and graft survival after use of $\mathrm{HCV}+$ donors for HT, the data on other transplant associated outcomes like rejection and CAV have been more limited. Although several of the aforementioned single-center studies and registries reported no differences in these outcomes [20, 24•, 25•, 26•], a single-center report by Gidea and colleagues reported that among 50 HT recipients in the DAAT era, including 22 with NAT + donors and 28 with NAT - donors, recipients of NAT + hearts had a significantly higher rate of treated acute cellular rejection (ACR) at 60 and 180 days, as well as a lower median time to occurrence of first rejection event [27]. The small numbers of patients in each arm as well as the retrospective nature of the study are important caveats in the interpretation of these results, and this area is deserving of further study.

Equally deserving of further study is the impact that dtHCV may have on development and progression of CAV. In the pre-DAAT era, reports suggested that $\mathrm{HCV}+$ donor status adversely affected CAV incidence and progression [13]. In the "new era," the influence of dt-HCV remains unclear, although two studies have shed light on this question, albeit to a limited degree. In Vanderbilt's report of $80 \mathrm{HCV}+$ heart transplants, there did appear to be a small increase in CAV prevalence in the HCV + group, although this difference was not statistically significant [24•]. Madan and colleagues compared 1-year prevalence of CAV among 6,943 recipients of $\mathrm{HCV}$ negative donors, 107 recipients of HCV NAT + donors, and 69 recipients of HCV $\mathrm{Ab}+\mathrm{NAT}-$ donors using data from the UNOS registry [28]. The rate of CAV was similar across all three groups in unadjusted and adjusted regression analyses, as well as in a propensity score matched analysis. The relatively smaller comparison groups, along with lack of information on CAV diagnostic methodology, DAAT use, and immunosuppression make it difficult to draw definitive conclusions from these data.

\section{Utilization of Hepatitis C Donor Hearts}

In the wake of the evidence to date, an increasing number of transplant centers have started to perform HT using HCV + donors. In 2018, 137 NAT + hearts were transplanted, compared to only 1 in 2015 [26•]. And although a UNOS analysis reported that only $12 \%$ of available $\mathrm{HCV}+$ hearts were recovered in 2019 [29], data suggests that once $\mathrm{HCV}+$ hearts are recovered they are more likely to be transplanted in the current era than was previously the case [25•], and that utilization rates of these hearts are approaching those of $\mathrm{HCV}-$ hearts [25•, 30]. Nonetheless, geographic disparities in the utilization of $\mathrm{HCV}+$ hearts persist [31], suggesting an opportunity for many centers to capitalize on this strategy. For centers seeking to begin HT using HCV + donors, recognition of the importance of a multidisciplinary approach is critical to ensure optimal patient outcomes. The multidisciplinary team should include, among others, transplant cardiologists and surgeons, expert consultants in hepatology or infectious disease, and transplant pharmacists. Equally important is input from and consideration of key stakeholders including hospital administration, payers, clinical investigators and, most importantly, the patient (Fig. 3).

\section{Cost and Cost-Effectiveness of HCV + Heart Transplants}

Given the high costs of DAAT juxtaposed against increasing evidence supporting the safety of $\mathrm{HCV}+\mathrm{HT}$, an important question at the individual, institutional, and societal level is whether use of $\mathrm{HCV}+$ transplants is a cost-effective strategy. The wholesale acquisition cost of a 12-week course of DAAT ranges anywhere from $\$ 83,000$ to $\$ 147,000$ according to 2016 estimates [32]. Logan and colleagues performed a cost-effectiveness analysis using a hypothetical scenario in which waitlisted candidates on inotropic or LVAD support were only willing to accept a HCV - heart vs. willing to accept any heart (HCV - or $\mathrm{HCV}+$ ) [33]. Relative to patients only willing to accept HCV - hearts, patients who accepted any heart gained 0.14 life-years of survival and 0.11 quality adjusted lifeyears (QALYs) largely due to shorter waitlist times, at a higher cost of $\$ 9418$ per patient (mainly due to DAAT costs). The incremental cost-effective ratio (ICER) was $\$ 85,602$ per QALY gained, thereby making this a costeffective strategy by standard cost-effectiveness metrics. As market competition drives down DAAT costs over time, and to the degree that utilization of $\mathrm{HCV}+$ donor hearts expands the donor pool and reduces waitlist times, we anticipate this strategy to prove itself increasingly costeffective in years to come.

\section{Society Consensus Statement for Use of HCV + Hearts for Transplantation}

The International Society for Heart Lung Transplantation (ISHLT) recently issued an expert consensus statement addressing the utilization of $\mathrm{HCV}$-infected organ donors in cardiothoracic transplantation [5•]. This document summarizes many of the commonly used DAAT, outlines potential drug-drug interactions that require consideration, and details differences and recommendations with regards to the aforementioned treatment strategies 


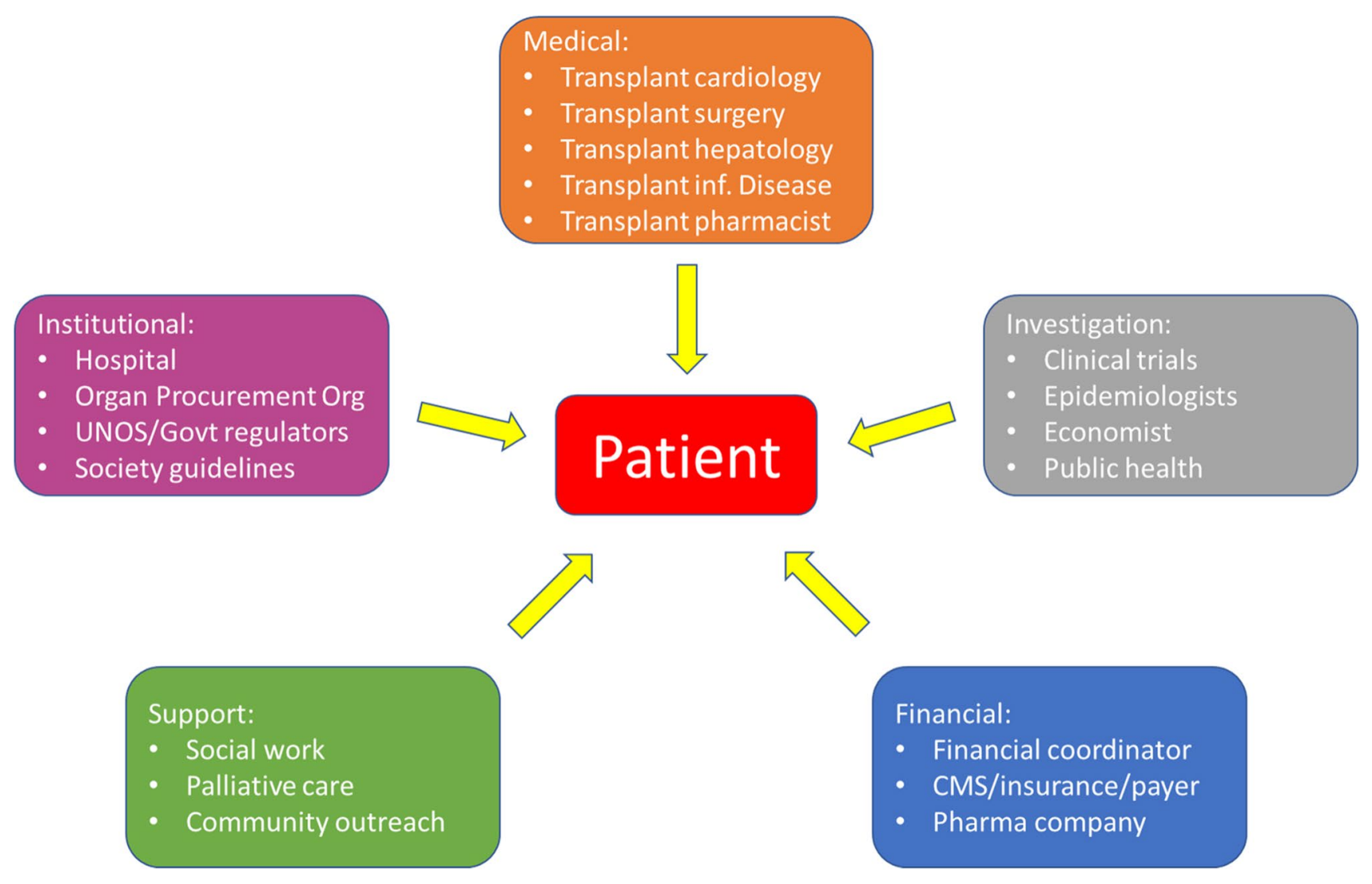

Fig. 3 A multidisciplinary approach to $\mathrm{HCV}+\mathrm{HT}$. Legend: Heart transplantation using $\mathrm{HCV}$ viremic donors requires a multidisciplinary team to assist with patient selection and support to optimize post-transplant outcomes. With the patient at the center, the multi-

(prophylactic vs. preemptive). Importantly, neither treatment strategy has been proven superior based on data available to date [20-23, 24•, 34-37]. The consensus statement emphasizes the importance of patient education and shared decision-making when considering $\mathrm{HCV}+$ donors, as well as ethical considerations and the critical role of a multidisciplinary team (Fig. 3).

\section{Outstanding Questions and Future Directions}

While the use of HCV + donors in HT has evolved rapidly over the past 6 years, important questions remain to be answered. What ramifications, if any, does dtHCV have on long-term allograft function, CAV, and development of donor specific antibodies? Are there non-cardiac ramifications of dt-HCV that remain to be fully elucidated? For patients who undergo HT with HCV NAT + donors, what is the ideal timing and duration of DAAT? And what role do HCV + donors play disciplinary team must include medical and institutional teams, along with defined support mechanisms for financial and emotional/psychological help

for patients awaiting multiorgan transplant? Finally, as HCV therapies continue to improve, is it possible to treat $\mathrm{HCV}$ in the donor heart prior to implantation in recipients? These and other questions must be systematically interrogated in ongoing registries and future multicenter trials.

\section{Conclusion}

In a "new era" marked by increasing numbers of $\mathrm{HCV}+$ deceased donors and significant advances in $\mathrm{HCV}$ treatment, utilization of $\mathrm{HCV}+$ donors for HT has afforded a novel strategy to expand the donor pool and reduce waitlist times. Short-term outcomes from multiple small single-center studies and registries have demonstrated the safety and efficacy of this strategy to date. We anticipate that longer term, more granular outcomes in larger numbers of patients will be forthcoming and will continue to shed light on this important topic in HT. 


\section{References}

Papers of particular interest, published recently, have been highlighted as: • Of importance

1. Virani SS, Alonso A, Aparicio HJ, Benjamin EJ, Bittencourt MS, Callaway CW, et al. Heart disease and stroke statistics-2021 update: a report from the American Heart Association. Circulation [Internet]. 2021 [cited 2021 Jul 5];143. Available from: https://www.ahajournals.org/doi/10.1161/CIR.00000 00000000950. Accessed 4 July 2021

2. Viral hepatitis surveillance report 2019 [Internet]. U.S. Department of Health and Human Services; 2021 Jul. Available from: https://www.cdc.gov/hepatitis/statistics/2019surveillance/HepC. htm. Accessed 4 July 2021

3. Hedegaard H, Miniño A, Warner M. Drug overdose deaths in the united states, 1999-2019. [Internet]. Hyattsville, MD: National Center for Health Statistics; 2020 Dec. Available from: https:// www.cdc.gov/nchs/products/databriefs.htm. Accessed 4 July 2021

4. Faust JS, Du C, Mayes KD, Li S-X, Lin Z, Barnett ML, et al. Mortality from drug overdoses, homicides, unintentional injuries, motor vehicle crashes, and suicides during the pandemic, March-August 2020. JAMA. 2021;326:84.

5. Aslam S, Grossi P, Schlendorf KH, Holm AM, Woolley AE, Blumberg E, et al. Utilization of hepatitis C virus-infected organ donors in cardiothoracic transplantation: an ISHLT expert consensus statement. The Journal of Heart and Lung Transplantation. 2020;39:418-32. This International Society of Heart Lung Transplantation expert consensus statement provides an important reference on key considerations when utilizing $\mathrm{HCV}+$ donor organs in HT, including treatment considerations and recommendations, and important drug-drug interactions.6. Strader DB, Seeff LB. A brief history of the treatment of viral hepatitis C: a history of HCV. Clinical Liver Disease. 2012;1:6-11.

6. Strader DB, Seeff LB. A brief history of the treatment of viral hepatitis C: a history of HCV. Clinical Liver Disease. 2012;1:6-11.

7. Feld JJ, Jacobson IM, Hézode C, Asselah T, Ruane PJ, Gruener $\mathrm{N}$, et al. Sofosbuvir and velpatasvir for HCV genotype 1, 2, 4, 5, and 6 infection. N Engl J Med. 2015;373:2599-607.

8. Foster GR, Afdhal N, Roberts SK, Bräu N, Gane EJ, Pianko S, et al. Sofosbuvir and velpatasvir for HCV genotype 2 and 3 infection. N Engl J Med. 2015;373:2608-17.

9. Afdhal N, Zeuzem S, Kwo P, Chojkier M, Gitlin N, Puoti M, et al. Ledipasvir and sofosbuvir for untreated HCV genotype 1 infection. N Engl J Med. 2014;370:1889-98.

10. AASLD-IDSA HCV Guidance Panel, Chung RT, Ghany MG, Kim AY, Marks KM, Naggie S, et al. Hepatitis C guidance, update: AASLD-IDSA recommendations for testing, managing, and treating hepatitis $\mathrm{C}$ virus infection. Clin Infect Dis. 2018;2018(67):1477-92.

11. Pfau PR, Rho R, DeNofrio D, Loh E, Blumberg EA, Acker MA, et al. Hepatitis $\mathrm{C}$ transmission and infection by orthotopic heart transplantation. J Heart Lung Transplant. 2000;19:350-4.

12. Ong JP, Barnes DS, Younossi ZM, Gramlich T, Yen-Lieberman B, Goormastic M, et al. Outcome of de novo hepatitis $\mathrm{C}$ virus infection in heart transplant recipients. Hepatology. 1999;30:1293-8.

13. Haji SA, Starling RC, Avery RK, Mawhorter S, Tuzcu EM, Schoenhagen $\mathrm{P}$, et al. Donor hepatitis-C seropositivity is an independent risk factor for the development of accelerated coronary vasculopathy and predicts outcome after cardiac transplantation. J Heart Lung Transplant. 2004;23:277-83.
14. Gasink LB, Blumberg EA, Localio AR, Desai SS, Israni AK, Lautenbach E. Hepatitis $C$ virus seropositivity in organ donors and survival in heart transplant recipients. JAMA. 2006;296:1843.

15. Goldberg DS, Abt PL, Blumberg EA, Van Deerlin VM, Levine M, Reddy KR, et al. Trial of transplantation of HCVinfected kidneys into uninfected recipients. N Engl J Med. 2017;376:2394-5.

16. Abdelbasit A, Hirji A, Halloran K, Weinkauf J, Kapasi A, Lien D, et al. Lung transplantation from hepatitis $\mathrm{C}$ viremic donors to uninfected recipients. Am J Respir Crit Care Med. 2018;197:1492-6.

17. Kwong AJ, Wall A, Melcher M, Wang U, Ahmed A, Subramanian A, et al. Liver transplantation for hepatitis $\mathrm{C}$ virus (HCV) non-viremic recipients with $\mathrm{HCV}$ viremic donors. Am J Transplant. 2019;19:1380-7.

18. Gottlieb RL, Sam T, Wada SY, Trotter JF, Asrani SK, Lima B, et al. Rational heart transplant from a hepatitis $\mathrm{C}$ donor: new antiviral weapons conquer the Trojan horse. J Cardiac Fail. 2017;23:765-7.

19. Schlendorf KH, Zalawadiya S, Shah AS, Wigger M, Chung CY, Smith S, et al. Early outcomes using hepatitis C-positive donors for cardiac transplantation in the era of effective direct-acting anti-viral therapies. J Heart Lung Transplant. 2018;37:763-9.

20. Woolley AE, Singh SK, Goldberg HJ, Mallidi HR, Givertz MM, Mehra MR, et al. Heart and lung transplants from HCV-infected donors to uninfected recipients. N Engl J Med. 2019;380:1606-17.

21. Bethea ED, Gaj K, Gustafson JL, Axtell A, Lebeis T, Schoenike $\mathrm{M}$, et al. Pre-emptive pangenotypic direct acting antiviral therapy in donor HCV-positive to recipient HCV-negative heart transplantation: an open-label study. The Lancet Gastroenterology \& Hepatology. 2019;4:771-80.

22. Aslam S, Yumul I, Mariski M, Pretorius V, Adler E. Outcomes of heart transplantation from hepatitis $\mathrm{C}$ virus-positive donors. J Heart Lung Transplant. 2019;38:1259-67.

23. McLean RC, Reese PP, Acker M, Atluri P, Bermudez C, Goldberg LR, et al. Transplanting hepatitis $\mathrm{C}$ virus-infected hearts into uninfected recipients: a single-arm trial. Am J Transplant. 2019;19:2533-42.

24. Schlendorf KH, Zalawadiya S, Shah AS, Perri R, Wigger M, Brinkley DM, et al. Expanding heart transplant in the era of direct-acting antiviral therapy for hepatitis C. JAMA Cardiol. 2020;5:167. Largest single-center outcomes report in HCV NAT+ HT in the contemporary era, demonstrating short-term safety and efficacy of pre-emptive DAAT.

25. Madan S, Patel SR, Rahgozar K, Saeed O, Murthy S, Vukelic S, et al. Utilization rates and clinical outcomes of hepatitis $\mathrm{C}$ positive donor hearts in the contemporary era. The Journal of Heart and Lung Transplantation. 2019;38:907-17. UNOS registry analysis demonstrating rising utilization rates of $\mathrm{HCV}+$ hearts, with acceptable short-term clinical outcomes after HCV+ HT.

26.• Kilic A, Hickey G, Mathier M, Sultan I, Gleason TG, Horn $\mathrm{E}$, et al. Outcomes of adult heart transplantation using hepatitis C-positive donors. JAHA [Internet]. 2020 [cited $2021 \mathrm{Jul}$ 3];9. Available from: https://www.ahajournals.org/doi/10.1161/ JAHA.119.014495. Largest UNOS registry analysis of HCV+ HT outcomes in the DAAT era, demonstrating good outcomes with 1-year follow-up. Accessed 4 July 2021

27. Gidea CG, Narula N, Reyentovich A, Fargnoli A, Smith D, Pavone J, et al. Increased early acute cellular rejection events in hepatitis C-positive heart transplantation. J Heart Lung Transplant. 2020;39:1199-207.

28. Madan S, Patel SR, Jorde UP. Cardiac allograft vasculopathy and secondary outcomes of hepatitis C-positive donor 
hearts at 1 year after transplantation. J Heart Lung Transplant. 2020;39:1318-21.

29. Moayedi Y, Fan CPS, Gulamhusein AF, Manlhiot C, Ross HJ, Teuteberg JJ, et al. Current use of hearts from hepatitis $\mathrm{C}$ viremic donors: a United Network for Organ Sharing registry analysis. Circ: Heart Failure [Internet]. 2018 [cited 2021 Jul 3];11. Available from: https://www.ahajournals.org/doi/10.1161/CIRCH EARTFAILURE.118.005276. Accessed 4 July 2021

30. Prakash K, Wainana C, Trageser J, Hahn A, Lay C, Pretorius V, et al. Local and regional variability in utilization and allocation of hepatitis C virus-infected hearts for transplantation. Am J Transplant. 2020;20:2867-75.

31 Li SS, Osho A, Moonsamy P, D’Alessandro DA, Lewis GD, Villavicencio MA, et al. Trends in the use of hepatitis $\mathrm{C}$ viremic donor hearts. J Thorac Cardiovasc Surg. 2020;16:S0022522320326404.

32. Rosenthal ES, Graham CS. Price and affordability of direct-acting antiviral regimens for hepatitis $\mathrm{C}$ virus in the United States. Infect Agents Cancer. 2016;11:24.

33. Logan C, Yumul I, Cepeda J, Pretorius V, Adler E, Aslam S, et al. Cost-effectiveness of using hepatitis $\mathrm{C}$ viremic hearts for transplantation into HCV-negative recipients. Am J Transplant. 2021;21:657-68.

34. Feld JJ, Cypel M, Kumar D, Dahari H, Pinto Ribeiro RV, Marks $\mathrm{N}$, et al. Short-course, direct-acting antivirals and ezetimibe to prevent $\mathrm{HCV}$ infection in recipients of organs from $\mathrm{HCV}$ infected donors: a phase 3, single-centre, open-label study. The Lancet Gastroenterology \& Hepatology. 2020;5:649-57.

35. Reyentovich A, Gidea CG, Smith D, Lonze B, Kon Z, Fargnoli $\mathrm{A}$, et al. Outcomes of the treatment with glecaprevir/pibrentasvir following heart transplantation utilizing hepatitis $\mathrm{C}$ viremic donors. Clin Transplant [Internet]. 2020 [cited 2021 Jul 4];34. Available from: https://onlinelibrary.wiley.com/doi/10.1111/ctr. 13989. Accessed 4 July 2021

36. Kapila N, Menon KVN, Al-Khalloufi K, Vanatta JM, Murgas C, Reino D, et al. Hepatitis C virus NAT-positive solid organ allografts transplanted into hepatitis $\mathrm{C}$ virus-negative recipients: a real-world experience. Hepatology. 2020;72:32-41.

37. Morris KL, Adlam JP, Padanilam M, Patel A, Garcia-Cortes R, Chaudhry S, et al. Hepatitis C donor viremic cardiac transplantation: a practical approach. Clin Transplant [Internet]. 2020 [cited $2021 \mathrm{Jul}$ 4];34. Available from: https://onlinelibrary.wiley.com/ doi/abs/10.1111/ctr.13764. Accessed 4 July 2021

Publisher's note Springer Nature remains neutral with regard to jurisdictional claims in published maps and institutional affiliations. 